\title{
A GENETICAL SYSTEM ADMITTING OF TWO DISTINCT STABLE EQUILIBRIA UNDER NATURAL SELECTION
}

\author{
A. R. G. OWEN \\ Department of Genetics, Cambridge
}

IT has long been known that in a population where different genotypes possess different selective advantages, it is possible for balanced polymorphism to exist. That is to say there is an equilibrium between the proportions of different genotypes, which is stable under random mating and differential viability and fertility. I do not know, however, whether any case has been recognised in nature or discussed theoretically in which it is possible for two distinct non-trivial stable equilibria to exist. (A trivial equilibrium is an extreme case when, for instance, some genes or genotypes are unrepresented in the population, and is of little interest.) It may therefore be of some theoretical interest to construct a genetical system admitting of two distinct stable states of balanced polymorphism.

Analogy with electrical and mechanical oscillating systems suggests that the necessary degree of complexity may be achieved by a system composed of two "coupled" sub-systems, possessing sufficiently unlike properties. Now the random mating of the sexes can be regarded as an interaction between two sub-populations. It is therefore natural to enquire whether a population in which the same genotypes have different viabilities in the two sexes is one admitting of a multiplicity of distinct equilibria. This expectation is verified. It is easy to specify a theoretical population involving three genotypes $\mathrm{AA}, \mathrm{A} a$ and $a a$ with selective advantages different within and between sexes, which has three equilibria, of which two are stable and the third unstable. For example with the scheme of viabilities

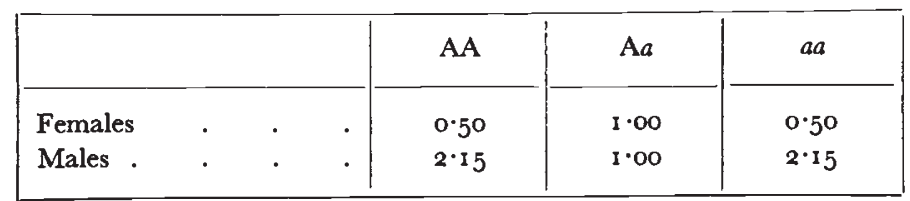

we obtain three equilibria characterised as follows for gene ratios in the two sexes and for stability.

\begin{tabular}{|c|c|c|l|}
\hline \multirow{2}{*}{$\begin{array}{c}\text { Equilibrium } \\
\text { state }\end{array}$} & \multicolumn{2}{|c|}{ Gene ratio $(\mathrm{A}: a)$} & Character \\
\cline { 2 - 4 } & In females & In males & \\
\hline I & 0.582 & 0.339 & Stable \\
II & $1 \cdot 000$ & Unstable \\
III & $1 \cdot 718$ & $2 \cdot 951$ & Stable \\
\hline
\end{tabular}

97 
I give herewith a brief discussion of systems of this type. It is seen that they may possess

(i) no non-trivial equilibrium state, or

(ii) a unique non-trivial equilibrium which may or may not be stable, or

(iii) three non-trivial equilibria, of which as many as two may be stable, the third being unstable.

All the possibilities relating to the case of three equilibria, and the necessary conditions on the viabilities have not been exhaustively explored numerically, but their complete examination would present no great difficulty. The analysis suggests that a necessary condition for the existence of three equilibria is that in at least one sex, at least one homozygote must be at an advantage relative to the heterozygote. It suggests further that the difference in viability between homozygotes and the heterozygote is required to be in opposite senses in the two sexes.

Assume random mating and the gene frequencies

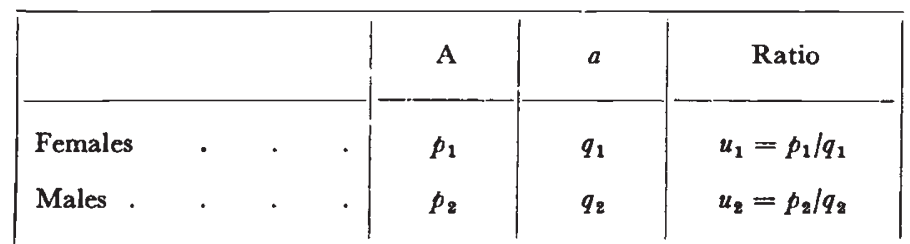

together with parameters to express selective advantage as follows

\begin{tabular}{|c|c|c|c|c|c|c|}
\hline & & & & AA & $\mathrm{A} a$ & $a a$ \\
\hline Females & & & . & $I-S_{1}$ & I & $\mathrm{I}-s_{1}$ \\
\hline Males . & . & . & . & $\mathrm{I}-\mathrm{S}_{2}$ & I & $\mathrm{I}-s_{1}$ \\
\hline
\end{tabular}

Then the genotypic proportions at an assigned phase in the life cycle are

\begin{tabular}{|c|c|c|c|}
\hline & $\mathrm{AA}$ & $\mathrm{A} a$ & $a a$ \\
\hline Females & $\left(1-S_{1}\right) p_{1} p_{2}$ & $p_{1} q_{2}+p_{2} q_{1}$ & $\left(1-s_{1}\right) q_{1} q_{2}$ \\
\hline Males & $\left(\mathrm{I}-\mathrm{S}_{2}\right) p_{1} p_{2}$ & $p_{1} q_{2}+p_{2} q_{1}$ & $\left(1-s_{2}\right) q_{1} q_{2}$ \\
\hline
\end{tabular}

Thus in the next generation

$$
\begin{aligned}
& u_{1}^{\prime}=\frac{\left(\mathrm{I}-\mathrm{S}_{1}\right) u_{1} u_{2}+\frac{1}{2}\left(u_{1}+u_{2}\right)}{\left(\mathrm{I}-s_{1}\right)+\frac{1}{2}\left(u_{1}+u_{2}\right)} \\
& u_{2}^{\prime}=\frac{\left(\mathrm{I}-\mathrm{S}_{2}\right) u_{1} u_{2}+\frac{1}{2}\left(u_{1}+u_{2}\right)}{\left(\mathrm{I}-s_{2}\right)+\frac{1}{2}\left(u_{1}+u_{2}\right)}
\end{aligned}
$$


In equilibrium $u_{1}^{\prime}=u_{1}, u_{2}^{\prime}=u_{2}$. Setting $t=u_{2} / u_{1}$ it is found that the equilibrium ratios are given by

$$
u_{1}=\frac{t-c_{1}}{\mathrm{I}-\mathrm{C}_{1} t}, \quad u_{2}=\frac{c_{2} t-\mathrm{I}}{\mathrm{C}_{2} t-t^{2}} \quad . \quad .
$$

where

$$
\begin{aligned}
c_{1} & =\mathrm{I}-2 s_{1} & c_{2} & =\mathrm{I}-2 s_{2} \\
\mathrm{C}_{1} & =\mathrm{I}-2 \mathrm{~S}_{1} & \mathrm{C}_{2} & =\mathrm{I}-2 \mathrm{~S}_{2}
\end{aligned}
$$

and $t$ satisfies the cubic equation

with

$$
f(t)=t^{3}-3 \alpha t^{2}+3 \beta t-\mathrm{I}=\mathrm{o}
$$

$$
\alpha=\frac{1}{3}\left(\mathrm{C}_{1} c_{2}+c_{1}+\mathrm{G}_{2}\right), \beta=\frac{1}{3}\left(\mathrm{C}_{2} c_{1}+c_{2}+\mathrm{G}_{1}\right)
$$

Using the facts that $f(0)=\mathrm{I}$, and that $f(t) \rightarrow \infty$ as $t \rightarrow \infty$, and considering maxima and minima of $f(t)$ it is found that $f(t)=0$ has a unique positive real root, unless the following conditions are satisfied simultaneously :-

and

$$
\left.\begin{array}{l}
\alpha>0, \alpha^{2}>\beta>0, \\
4 \alpha^{3}+4 \beta^{3}-3 \alpha^{2} \beta^{2}-6 \alpha \beta+1<0
\end{array}\right\} . . \quad .
$$

When these conditions are fulfilled there are three distinct real positive values, $t_{1}, t_{2}, t_{3}$ of $t$ satisfying $f(t)=0$. If also $u_{1}$ when calculated by (I) is positive for each of these values of $t$, then there are three distinct non-trivial equilibria. This latter requirement can be expressed by saying that $t_{1}, t_{2}$ and $t_{3}$ must lie within the interval whose termini are $t=c_{1}, t=\mathrm{I} / \mathrm{C}_{1}$.

Condition (2) may be written in the form

$$
\phi(\beta)=\beta^{3}-\frac{3}{4} \alpha^{2} \beta^{2}-\frac{3}{2} \alpha \beta+\left(\frac{1}{4}+\alpha^{3}\right)<0 .
$$

For given $\alpha$ and variable $\beta$ it is seen that $\phi(\beta)$ has a minimum value

$$
m=-2\left(\frac{1}{16} \alpha^{4}+\frac{1}{2} \alpha\right)^{\frac{3}{2}}+\mathrm{I}+\frac{5}{8} \alpha^{3}-\frac{1}{32} \alpha^{6}
$$

achieved when

$$
\beta=\beta_{m}=\frac{1}{4} \alpha^{2}+\sqrt{\frac{1}{16} \alpha^{2}+\frac{1}{2} \alpha}
$$

The table of values of $m$ and $\beta_{m}$ shows that for all values of $\alpha$ in excess of $\alpha_{0}$ (where $\alpha_{0}$ is about $\mathrm{I}_{5}$ ), it is possible to find a range of values of $\beta$ in the neighbourhood of $\beta_{m}$ for which all the conditions for three equilibria are satisfied.

There is however a second continuum of $\alpha, \beta$ values satisfying the conditions. For taking $\beta=\alpha$ we see that the equation

has the roots

$$
t^{3}-3 \alpha t+3 \alpha t-\mathrm{I}=0
$$

$$
t_{1}=\frac{1}{2}\left(k-\sqrt{k^{2}-4}\right), \quad t_{2}=\mathrm{I}, \quad t_{3}=\frac{1}{2}\left(k+\sqrt{k^{2}-4}\right)
$$

where $k=3 \alpha-\mathrm{I}$. Thus there are three equilibria provided that

$$
3 \alpha-\mathrm{I}=k>2 \text {, }
$$


i.e. provided that $\alpha>$ I. The same applies for all values of $\alpha$ greater than unity, and for values of $\beta$ sufficiently close to $\alpha$. It appears therefore that there are two distinct viability ranges for which the system has three equilibria.

TABLE

\begin{tabular}{|c|c|c|c|}
\hline$a$ & $\phi_{m}$ & $\beta_{m}$ & $a^{2}$ \\
\hline $1 \cdot 0$ & 0.00000 & $1 \cdot 000$ & $1 \cdot 00$ \\
$1 \cdot 2$ & 0.18784 & $1 \cdot 214$ & $1 \cdot 44$ \\
$1 \cdot 4$ & 0.21827 & $1 \cdot 424$ & $1 \cdot 96$ \\
$1 \cdot 5$ & 0.00013 & $1 \cdot 595$ & $2 \cdot 25$ \\
$1 \cdot 6$ & -0.05549 & $1 \cdot 740$ & $2 \cdot 56$ \\
$1 \cdot 8$ & -0.31425 & $2 \cdot 057$ & 3.24 \\
$2 \cdot 0$ & $-1 \cdot 90684$ & $2 \cdot 414$ & $4 \cdot 00$ \\
$\infty$ & $-\infty$ & $\infty$ & $\infty$ \\
\hline
\end{tabular}

It is informative to consider the special case when in each sex the homozygotes have equal viabilities. Take therefore $\mathrm{S}_{1}=s_{1}$, $\mathrm{S}_{2}=s_{2}$. Then

so that

$$
\alpha=\mathrm{I}+\frac{4}{3}\left(s_{1} s_{2}-s_{1}-s_{2}\right),
$$

$$
\left(\mathrm{I}-s_{1}\right)\left(\mathrm{I}-s_{2}\right)=\mathrm{I}+\frac{3}{4}(\alpha-\mathrm{I}) .
$$

Since $\alpha>1$ is a necessary condition for three equilibria we get as a further necessary condition

$$
\left(\mathrm{I}-s_{1}\right)\left(\mathrm{I}-s_{2}\right) \geqslant \mathrm{I} \text {. }
$$

Consequently at least one of the homozygous viabilities $\mathrm{I}-s_{1}, \mathrm{I}-s_{2}$ exceeds unity.

The more specialised case in which $\mathrm{S}_{1}=s_{1}=\mathrm{S}_{2}=s_{2}$, gives all viabilities equal as between sexes, a situation which admits of at most one equilibrium. This suggests that for three equilibria to exist, the difference in viability between homozygotes and heterozygotes is required to be in opposite senses in the two sexes.

Returning to the general case, we note the following procedure for investigating the stability of any equilibrium state. In place of $u_{1}$ and $u_{2}$, write $u_{1}+x_{1}, u_{2}+x_{2}$ giving $u_{1}$ and $u_{2}$ their equilibrium values. Then neglecting quadratic terms in $x_{1}$ and $x_{2}$ we get the approximate recurrence relations

where

$$
x_{1}^{\prime}=b_{11} x_{1}+b_{12} x_{2}, \quad x_{2}^{\prime}=b_{21} x_{1}+b_{22} x_{2},
$$

$$
b_{i j}=u_{i} \frac{\partial}{\partial u_{j}}\left(\log u_{i}^{\prime}\right) \quad(i, j=1,2)
$$

and $u_{1}, u_{2}$ are put equal to equilibrium values after differentiation. The equations in $x_{1}, x_{2}$ have solutions of the form

$$
x_{1 n}=\mathrm{B}_{11} \lambda_{1}{ }^{n}+\mathrm{B}_{12} \lambda_{2}{ }^{n}, \quad x_{2 n}=\mathrm{B}_{21} \lambda_{1}{ }^{n}+\mathrm{B}_{22} \lambda_{2}{ }^{n}
$$


where $\lambda_{1}, \lambda_{2}$ are the latent roots of the matrix $\left(b_{i j}\right)$. Consequently the equilibrium state is stable or unstable according as to whether both $\lambda_{1}, \lambda_{2}$ are or are not less than unity.

The example given in the introduction was constructed by taking $\alpha=\mathbf{I} \cdot \mathbf{I}=\beta$, so that the appropriate roots were

$$
t_{1}=0.582 \mathrm{Iog}, \quad t_{2}=\mathrm{I} \cdot 0, \quad t_{3}=\mathrm{I} \cdot 7 \mathrm{I} 7,89 \mathrm{I} .
$$

We require as subsidiary conditions either

(i) $\mathrm{I}-2 s_{1}=c_{1}<0.582$ and $\mathrm{I}-2 \mathrm{~S}_{1}=\mathrm{C}_{1}<0.582$

corresponding to homozygous viabilities $\mathrm{I}-s_{1}, \mathrm{I}-\mathrm{S}_{1}$ less than 79 per cent., or

(ii) $\mathrm{I}-2 s_{1}=c_{1}>\mathrm{I} \cdot 7 \mathrm{I} 8$ and $\mathrm{I}-2 \mathrm{~S}_{1}=2 \mathrm{~S}_{1}=\mathrm{C}_{1}>\mathrm{I} \cdot 7 \mathrm{I} 8$

corresponding to homozygous viabilities greater than 136 per cent. For simplicity we take an extreme case in which $c_{1}=\mathrm{C}_{2}=0$, (i.e. the homozygotes among females have viabilities of $5^{\circ}$ per cent. only, while in the males their viabilities are 215 per cent.) and obtain three equilibria as described, the stability roots being :-

$$
\text { I. } 0.9617,0.0383 \text {; II. } 0 \text {, I } \cdot 0159 \text {; III. } 0.7277,0.1283 \text {. }
$$

As a second example we may take the table of viabilities

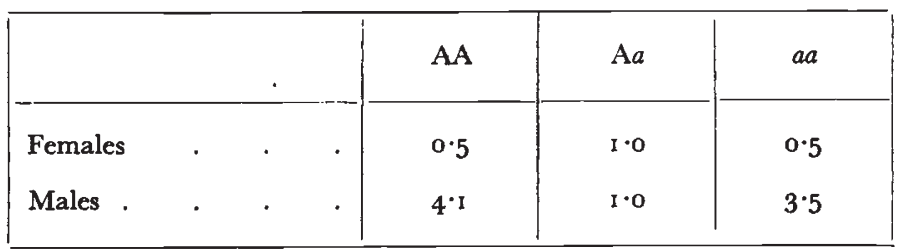

\begin{tabular}{|c|c|c|c|c|}
\hline \multirow{2}{*}{ State } & \multicolumn{2}{|c|}{ Gene ratio $(\mathrm{A}: a)$} & \multirow{2}{*}{ Character } & \multirow{2}{*}{$\begin{array}{l}\text { Stability } \\
\text { roots }\end{array}$} \\
\hline & In females & In males & & \\
\hline I & $0 \cdot 16$ & 0.026 & Stable & $0.91, \quad 0.07$ \\
\hline II & $x \cdot 42$ & $2 \cdot 00$ & Unstable & $1.02,-0.14$ \\
\hline III & $44^{2}$ & 19.57 & Stable & 0.92 \\
\hline
\end{tabular}

for which $\alpha=2$ and $\beta=2 \cdot 4$, obtaining equilibria as follows :-

Both examples illustrate the case when there are two equilibria, each characterised by a rather large disparity of the gene ratios in the two sexes. If such differences in gene ratio were to be observed in an ecological situation, they would be suggestive of the existence of the peculiar kind of balance that has been examined.

The present discussion clearly does not exclude the possibility of cases in which there are three non-trivial equilibria of which only 
one (presumably the intermediate one) is stable. An answer to this question would be of interest, and could take one of two forms. It may be possible to demonstrate algebraically that these cases do not exist. On the other hand it may be possible to construct an illustrative numerical example.

In the case where one allele $A$ is lethal in both sexes when in the homozygous condition AA it is easy to see that there are at most two non-trivial equilibria of which only one is stable.

The writer has profited from helpful discussions with Walter Peters at present working in this department, whose field observations suggested this problem.

\section{SUMMARY}

It is shown that different viabilities of genotypes in the two sexes may lead to either of two stable equilibrium states being realised in a population under random mating.

\section{REFERENCE}

OWEN, A. R. G. I952. A genetical system admitting of two stable equilibria. Nature I70, II 27. 